RU Маркеры аргументации в англоязычных научных статьях гуманитарного и естественно-научного направлений

\begin{abstract}
Некрасова М. Ю., Миронцева С. С., Байко В. А.
Аннотация. Цель исследования заключается в выявлении видов и особенностей реализации маркеров аргументации в англоязычных научных статьях естественно-научного и гуманитарного направлений. Научная новизна исследования определяется тем, что впервые рассмотрены типичные для англоязычного научного дискурса маркеры аргументации из разряда незнаменательной лексики. Приведен сопоставительный анализ функционирования маркеров аргументации в статьях естественнонаучного и гуманитарного направлений. В результате исследования были выделены следующие наиболее частотные типы маркеров: пояснение, вывод, обоснование, возражение, пример. Выявлено, что в статьях естественно-научного и гуманитарного направлений маркеры аргументации в большинстве случаев выполняют комментирующую, информативную, сигнализирующую и организационную функции, что объясняется спецификой исследуемых статей.
\end{abstract}

\title{
EN Argumentative Markers in the English-Language Scientific Humanitarian and Natural Scientific Discourse
}

\begin{abstract}
Nekrasova M. Y., Mirontseva S. S., Baiko V. A.
Abstract. The research objectives are as follows: to identify the types of argumentative markers in the English-language scientific humanitarian and natural scientific discourse, to reveal the specificity of their realization. Scientific originality of the study is conditioned by the fact that the authors for the first time examine argumentative markers-function words typical for English-language scientific discourse. The paper provides a comparative analysis of argumentative markers functioning in natural scientific and scientific humanitarian discourse. The conducted research allows identifying the most frequent types of argumentative markers: clarification, conclusion, justification, objection, example. It is shown that in natural scientific and scientific humanitarian discourse argumentative markers mostly perform commenting, informative, indicative and organizational functions, which is explained by the specificity of discourse under study.
\end{abstract}

\section{Введение}

Маркеры аргументации присутствуют в различных дискурсах и представляют собой языковые единицы, которые выполняют структурно-смысловую функцию при построении текста. Во избежание снижения уровня понимания фактической информации, изложенной автором, используются языковые единицы, не содержащие прямых сведений, но употребление которых запускает операцию по пониманию смысла текста. Данные языковые единицы направляют мысль интерпретатора по задуманному автором пути для следования за его мыслью.

Актуальность данного исследования обусловлена ростом популярности информационного пространства в условиях расширяющихся межкультурных контактов. Вследствие расширения научных горизонтов и повышения интереса к научным статьям обозначилась потребность их правильной интерпретации. Таким образом, возникает необходимость более углубленного изучения и описания функционирования маркеров аргументации как средств связности и логичности в современных англоязычных статьях научного дискурса.

Объектом исследования являются маркеры аргументации в научном дискурсе.

Предметом исследования являются виды маркеров аргументации и их реализация в англоязычных научных статьях гуманитарного и естественно-научного направлений.

Для выявления маркеров аргументации в англоязычных научных статьях гуманитарного и естественнонаучного направлений в настоящем исследовании были поставлены следующие задачи:

1) классифицировать виды маркеров аргументации в научных исследованиях; 
2) выявить функции маркеров аргументации в научных статьях;

3) провести сравнительную характеристику маркеров аргументации в англоязычных научных статьях гуманитарного и естественно-научного направлений.

Для решения поставленных задач в работе применены следующие методы исследования: методы контекстуального и лингвистического анализа, которые необходимы для интерпретации функционирования маркеров в научных статьях естественно-научного и гуманитарного направления; метод сопоставительного анализа, применяемый для сравнения функционирования видов маркеров аргументации; метод сплошной выборки языкового материала.

Основную теоретическую базу нашего исследования составили работы отечественных ученых, посвященные изучению семантических, структурных и прагматических свойств маркёров аргументации (Березина, 2018), типов и функций дискурсивных маркеров (Болсуновская, Зеремская, Дубровская, 2015; Когут, 2016); исследования в области теории аргументации (Костюшкина, Баребина, Колмогорова, 2013), стратегий аргументации в научном дискурсе (Нужнова, Бабаева, Жуковская, 2019). В качестве теоретической основы данного исследования выступает классификация типов маркеров аргументации и их функций, рассматриваемая П. А. Бороденковым (2009).

Практическая значимость работы состоит в возможности использования результатов и материалов исследования в практике преподавания курсов лексикологии современного английского языка, лингвистического анализа текста, при изучении научного дискурса.

\section{Основная часть}

Анализу подверглись маркеры аргументации в количестве 1520 единиц. Источником исследования послужили 20 англоязычных научных статей, из которых 10 гуманитарного направления и 10 естественно-научного. Тематика гуманитарных статей: лингвистика, когнитивная лингвистика, литература, музыка. Тематика естественно-научных статей: геология, философия, медицина и биология. Данные статьи были написаны в период с 2016 по 2021 г.

Маркеры аргументации представляют собой языковые единицы, которые выполняют структурно-смысловую функцию при построении дискурса (текста). Они пронизывают научный текст, обеспечивают грамматическую и семантическую цельность дискурса, организуют смысловую связность его единиц. Иными словами, сопровождают любую мысль, любое рассуждение автора, позволяют понять особенности его менталитета, составляют важный аспект научной картины мира учёного, создающего научный текст (Fraser, 2009; Schiffrin, 1987).

В работах отечественных ученых маркеры часто рассматриваются с позиции функционального подхода. В работах С. В. Когут (2016) на основании функционально-прагматического подхода маркеры составляют три группы:

1) маркеры структурной и смысловой связности научного текста;

2) маркеры мнения и оценки автора;

3) маркеры взаимодействия говорящего и слушающего (с. 102-103).

В то же время С. В. Когут (2014) определяет маркеры в научном дискурсе как ориентировочные сигналы, помечающие определенные смыслы в научной речи и выполняющие при этом определенные функции, являясь средством связи на уровне дискурса (с. 102).

В соответствии с этими аспектами исследователи предлагают ряд классификаций типов маркеров аргументации. Причем виды маркеров напрямую зависят от их функционирования в тексте. Например, Н. С. Березина (2018) выделяет три типа маркеров аргументации: подтверждение, опровержение, объяснение (с. 17). В свою очередь П. А. Бороденков (2009), опираясь на вышеупомянутую классификацию, предлагает более подробную, расширенную классификацию, основанную на структуре научной статьи и компонентах аргументации: обоснование, пояснение, определение, пример, развенчание, возражение, вывод (с. 15-17).

В ходе исследования на основе анализа англоязычных научных статей нами были выявлены маркеры аргументации в соответствии с классификацией П. А. Бороденкова в количестве 1105 единиц (72\% от общего числа анализируемых маркеров) в научных статьях гуманитарного направления и 415 единиц (28\% соответственно) в статьях естественно-научного направления.

В результате количественного анализа текстов данных статей можно сделать вывод о том, что к наиболее частотным маркерам аргументации в структуре статей естественно-научного и гуманитарного направления относятся:

1) пояснение ( $\approx 28 \%$ от выборки): furthermore, on average, overall, in addition, because, notably, more specifically, more generally, basically, actually, accordingly, in this way, especially, likewise, moreover, thereafter / кроме того, в среднем, в целом, в дополнение, потому что, в частности, более конкретно, в более общем плане, в основном, фактически, соответственно, таким образом, особенно, аналогично, кроме того, впоследствии;

2) вывод (₹23\%): thus, finally, to this end, overall, hence, in conclusion, subsequently, generally, to sum it up, consequently, so, all in all, in consequences, in general, to summaries, to conclude, after all, in sum, a summary, for a summary, overall / таким образом, в конечном итоге, с целью, в целом, следовательно, в заключение, впоследствии, в целом, подводя итог, следовательно, так, отметим следующее, в результате, в общем, резюмируя, подводя итог, в итоге, суммируя, заключая, в целом;

3) обоснование ( $19 \%)$ : nevertheless, also, however, moreover, subsequently, indeed, because, only, still, apparently, as before, whenever, evidently, perhaps, clearly, alternatively, duе to, typically / тем не менее, также, однако, более того, 
безусловно, потому что, только, все еще, по-видимому, как и прежде, во всех случаях, очевидно, возможно, несомненно, в противном случае, из-за, как правило;

4) возражение ( $\approx 15 \%)$ : on the other hand, on the one hand, at the same time, in other words, in the other half, in contrast, but, on the contrary, on the other side, from this moment, only, by contrast, when doing so, at least, yet, apart from this, despite the fact that, unfortunately, in comparison, whereas, although, almost, instead / с другой стороны, с одной стороны, в то же время, другими словами, в противном случае, в противоположность сказанному, но, напротив, с другой стороны, с этого момента, только, в сравнении, при этом, по крайней мере, пока, помимо этого, несмотря на то, что, к сожалению, в сравнении, тогда как, хотя, почти, вместо;

5) пример ( $\approx 10 \%)$ : for example, for instance, as a result of, similarly, first, second, the result of, secondly, firstly, to illustrate / например, к примеру, как результат, аналогично, во-первых, во-вторых, в результате, второе, прежде всего, чтобы проиллюстрировать.

Такие маркеры аргументации, как маркеры определения и развенчания составили незначительный про-

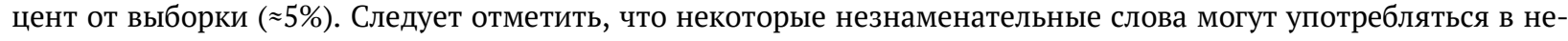
скольких типах исследуемых аргументативных речевых действий.

Функционирование маркеров аргументации в научном тексте довольно разнообразно. В широком смысле они выполняют прагматические, социальные, коммуникативные, текстообразующие (дискурсообразующие) функции. Кроме того, внутри каждой из этих сфер есть ряд своих функциональных аспектов. Важно отметить, что маркеры аргументации обладают широкой полифункциональностью. Они могут функционировать в научном тексте и как вспомогательные, речеорганизующие единицы, и как носители содержательной, фактуальной информации.

Согласно полученным данным для выражения аргументативного речевого действия маркеры аргументации в большинстве случаев выполняют следующие функции в текстах статей гуманитарного и естественнонаучного направлений:

1) комментирующую:

Modern [Indonesian] literature had already emerged in the Dutch East Indies long before the establishment of Balai Pustaka and before the invention of Indonesian nationalism through the use of political connotation from the word Indonesia which took place in the early of the twentieth century. In addition, literary critics agree that the Indonesian language is different from the Malay language (Nugraha, Suyitnno, 2019, с. 605). / Современная [индонезийская] литература появилась в Голландской Ост-Индии задолго до основания издательства Балей Пустака и задолго до приобретения словом «Индонезия» политической окраски благодаря индонезийскому национализму, возникшему в начале двадцатого века. Более того, литературные критики сходятся во мнении, что индонезийский язык отличается от малайского (здесь и далее перевод выполнен авторами статьи. - М. Н., С. М., В. Б.).

В данном примере речь идет о литературе, о появлении современной литературы и индонезийском национальном сознании. Автор для подтверждения своей мысли использует наречие in addition (более того), которое дает комментарий к основному сообщению, более точно поясняя разницу в индонезийском и малайском языках. Данный маркер пояснения помогает автору подкрепить свою мысль, говоря о том, что литературные критики сходятся во мнении об отличии малайского и индонезийского языка;

2) сигнализирующую:

While our findings do allow clear conclusions to be drawn, the study presents several limitations. Firstly, the small size of our sample, and especially the healthcare professionals subgroup, prevents us from extrapolating our data (Lacamper, Perez Pérez, Piñol-Ripoll, 2021, с. 36). / Хотя наши результаты позволяют сделать четкие выводы, исследование имеет несколько ограничений. Прежде всего, небольшой размер выборки нашего исследования, а именно подгруппы медицинских работников, не позволяет нам экстраполировать полученные данные.

В приведенном фрагменте говорится о результатах проведенного исследования, которое имеет ряд ограничений. Чтобы подробно описать ограничения и ввести их в качестве примера, автор использует маркер аргументации firstly (прежде всего). С помощью данного маркера автор эксплицитно указывает на наглядность, приводя пример того, что небольшой размер выборки и особенно подгруппы медицинских работников не позволяют исследователям экстраполировать их данные;

3) информативную:

In many languages, the word meaning "orange" is derived from the fruit Citrus sinensis, the word meaning "pink" is derived from roses, salmon, or peaches, and the word meaning "gray" is often derived from the word for ashes. Likewise, it is quite possible that monolexemic color terms are historically derived from concrete referents as well, but the etymological distance could be too great or the historical development of a language too poorly understood to link the referents to the color word (Johansson, Anikin, Aseyev, 2019, с. 6). / Во многих языках слово, означающее «оранжевый», происходит от фрукта апельсин, слово, означающее «розовый», происходит от роз, лосося или персиков, а слово, означающее «серый», часто происходит от слова, обозначающего пепел. Аналогичным образом вполне возможно предположить, что монолексемные цветовые термины также исторически произошли от конкретных референтов, однако слишком большая этимологическая дистанция или плохо понимаемое историческое развитие языка препятствуют связыванию референтов с цветовым словом.

В примере речь идет о происхождение значения слов «оранжевый», «розовый», «серый», которые исторически произошли от цвета фруктов, цветов, пепла, рыбы. Для пояснения того, что, возможно, монолексемные цветовые термины также произошли от конкретных референтов, автор использует маркер аргументации like- 
wise (аналогичным образом), который воздействует на читателя, отсылая его к изложенной ранее в тексте информации, и акцентирует внимание на достоверности информации, упоминающейся после данного маркера;

4) организационную:

For example, the most successful interior-point algorithm in practice is Mehrotra's Predictor-Corrector (MPC) algorithm which has not been proved to be polynomial although a lot of effort has been made. In fact, MPC may not be polynomial (Yang, 2021, с. 660). / Например, на практике наиболее успешным алгоритмом внутренней точки для линейного программирования является алгоритм предиктора-корректора Мехротры (МПК), полиномиальность которого не была доказана, хотя и были предприняты большие усилия. Если быть точным, то МПК может не быть полиномиальным.

В данном фрагменте маркер примера for example (например) употребляется автором для введения нового текстового фрагмента и акцентирования внимания читателя на информации, упоминающейся после него. В то же время маркер аргументации in fact (если быть точным) выступает в роли организующего сигнала, обеспечивающего переход от одного фрагмента к другому, одновременно являясь сигналом для читателя, акцентирующим его внимание на данной дополнительной информации.

В ходе анализа было выявлено, что функционирование маркеров напрямую зависит от их роли в научном тексте и, соответственно, разные типы маркеров буду преобладать в разных частях статьи. В основном маркеры обоснования встречаются во введении при постановке проблемы, маркеры пояснения, примера и возражения встречаются в основной части статьи, а маркеры вывода в выводе - для подведения итогов и завершения мысли.

В ходе исследования было выявлено, что основной функцией маркеров обоснования является постановка проблемы, у возражения - ввод контраргументов, функцией маркеров пояснения является подтверждение достоверности текста, маркеры примера несут в себе функцию конкретизации, ввода дополнительной информации, а маркеры вывода - функцию подведения итогов.

Как показал проведенный анализ в статьях гуманитарного направления, на 71047 слов встречается 1105 маркеров, что составляет $\approx 2 \%$ от общего количества слов. Статьи естественно-научного направления содержат 53822 слова, из которых 415 - маркеры аргументации, что составляет $\approx 0,8 \%$ от общего количества.

На Рисунке 1 нами предложена интенсивность использования маркеров аргументации в гуманитарных и естественно-научных статьях.

\section{Гуманитарные статьи}

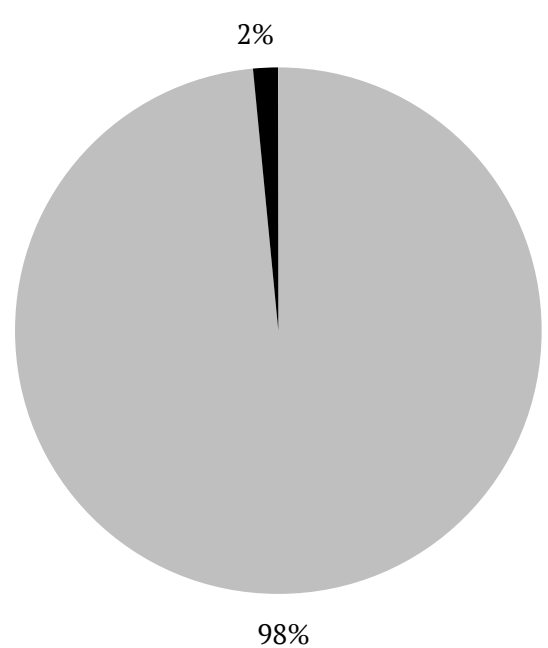

$\square$ Всего слов $\square$ Маркеров

\section{Естественно-научные статьи}

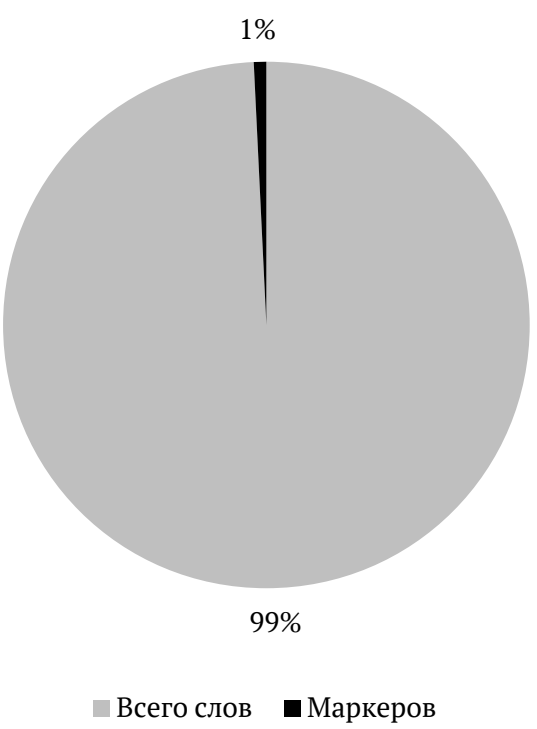

Рисунок 1. Соотношение количества слов и маркеров в англоязычных статьях гуманитарного и естественно-научного направления

Исходя из полученных данных, видно, что доля маркеров аргументации преобладает в гуманитарных статьях. Данный факт объясняется тем, что в гуманитарных статьях необходима большая связность текста, а в естественно-научных предпочтение отдается формулам, графикам, таблицам и их описанию.

В ходе проведенного анализа было выявлено в гуманитарных статьях 130 разновидностей маркеров, среди которых 14 маркеров обоснования, 26 маркеров пояснения, 22 - определения, 20 - примера, 19 маркеров возражения, 10 - развенчания и 19 маркеров вывода. В естественно-научных статьях нами было выделено 80 разновидностей маркеров, среди которых 12 маркеров обоснования, 18 маркеров пояснения, 12 - определения, 8 - примера, 11 маркеров возражения, 6 - развенчания и 13 маркеров вывода. Динамика распределения данных показателей отображена на Рисунке 2. 


\section{Маркеры аргументации}

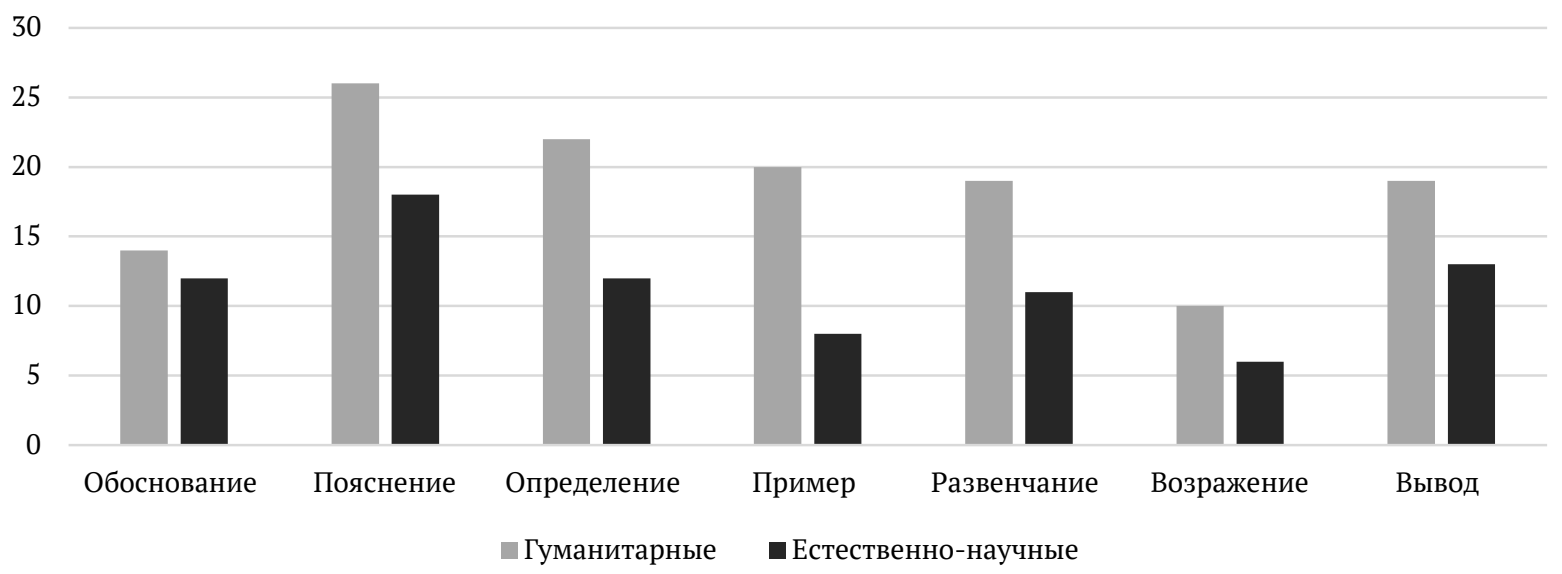

Рисунок 2. Сравнение маркеров аргументации

в англоязычных статьях гуманитарного и естественно-научного направления

На основе данных, представленных выше, можно заключить, что наиболее употребительными маркерами в проанализированных статьях оказались маркеры пояснения. Преобладание маркеров данного типа объясняется тем, что они напрямую отражают основные характеристики научного стиля: связность, ясность, точность, логичность и последовательность изложения. Проведенное исследование показало, что в гуманитарных статьях используются на втором месте по частотности употребления маркеры определения. Далее по шкале идут маркеры примера, а за ними примерно с равным процентным соотношением маркеры развенчания и вывода. В естественно-научных статьях на втором месте по частотности употребления маркеры обоснования и вывода. Далее идут маркеры определения и развенчания.

Анализ показал, что главная функция используемых маркеров аргументации в статьях обоих исследуемых нами направлений комментирующая и направлена на максимальное упрощение для читателя ориентации в тексте статьи. В то же время в гуманитарных статьях преобладают маркеры примера, выполняющие информативную функцию, а в естественно-научных - обоснования и вывода, выполняющие сигнализирующую и организационную функции. Данный факт объясняется тем, что гуманитарные науки изучают человека и его жизнедеятельность, а значит, только получив эмпирические данные, мы можем сделать выводы, в то время как естественные науки более абстрактны, и для того, чтобы провести какое-то исследование, требуется его обоснование с точки зрения законов природы.

\section{Заключение}

Проведенное нами исследование позволяет сделать выводы, что к наиболее частотным маркерам аргументации в структуре статей естественно-научного и гуманитарного направления относятся: пояснение ( $28 \%)$, вывод ( $\approx 23 \%)$, обоснование ( $\approx 19 \%)$, возражение ( $\approx 15 \%)$, пример ( $\approx 10 \%)$.

В ходе исследования было установлено, что в гуманитарных статьях к наиболее частотным маркерам относятся nevertheless, also, however, furthermore, on average, overall, in addition, for example, for instance, in contrast, on the other hand, thus, finally, generally; в то время как в естественно-научных статьях к наиболее частотным маркерам относятся indeed, however, also, moreover, because, for example, first, second, on the other hand, but, finally, to summary, hence, overall.

Результаты представленного исследования свидетельствуют о том, что в статьях гуманитарного направления маркеры аргументации составляют $\approx 2 \%$ от общего количества слов, а в естественно-научных статьях маркеры составляют $\approx 0,8 \%$.

В результате распределения типов маркеров аргументации можно сделать вывод, что самым частотным типом в естественно-научных и гуманитарных статьях являются маркеры пояснения ( $28 \%$ от всей выборки). На втором месте по частотности употребления в гуманитарных - маркеры определения, а в естественнонаучных - маркеры обоснования и вывода.

Опираясь на проведенный анализ, можно заключить, что в примерах с использованием маркеров аргументации в большинстве случаев маркеры выполняют комментирующую, информативную, сигнализирующую и организационную функции.

Перспективы дальнейшего исследования маркеров аргументации в англоязычных научных статьях гуманитарного и естественно-научного направлений видятся в изучении многокомпонентных маркеров, поскольку такие специальные лексические единицы обладают высокой степенью мотивированности, так как они передают необходимые признаки выражаемых ими понятий. 


\section{Источники | References}

1. Березина Н. С. Маркёры аргументации в научном тексте: семантика и функционирование (на материале научной прозы М. М. Бахтина, Б. В. Томашевского, Ю. Н. Тынянова): дисс. ... к. филол. н. Владивосток, 2018.

2. Болсуновская Л. М., Зеремская Ю. А., Дубровская Н. В. Виды дискурсивных маркеров в русскоязычных и англоязычных научных статьях // Вестник Томского государственного педагогического университета. 2015. № 4 (157)

3. Бороденков П. А. Маркеры аргументации в обиходном дискурсе: автореф. дисс. ... к. филол. н. Иваново, 2009.

4. Когут С. В. Дискурсивные маркеры в научном тексте: этнокультурный и дискурсивный аспекты (на русском и немецком языковом материале): дисс. ... к. филол. н. Томск, 2016.

5. Когут С. В. Дискурсивные маркеры как отражение своеобразия естественнонаучной и научно-гуманитарной картины мира // Филологические науки. Вопросы теории и практики. 2014. № 10 (40): в 3-Х ч. Ч. І.

6. Костюшкина Г. М., Баребина Н. С., Колмогорова А. В. Концептуальная систематика аргументации: коллективная монография. Иркутск: ИГЛУ, 2013.

7. Нужнова Е. Е., Бабаева Т. Б., Жуковская Н. В. Стратегия аргументации в научном дискурсе // Вестник Пермского национального исследовательского политехнического университета. Проблемы языкознания и педагогики. 2019. № 2.

8. Fraser B. An Account of Discourse Markers // International Review of Pragmatics. 2009. Vol. 1. Issue 2.

9. Johansson N., Anikin A., Aseyev N. Color sound symbolism in natural languages // Language and Cognition. 2019. № 12 (1).

10. Lacamper R., Perez Pérez R. M., Piñol-Ripoll G. Understanding of legal aspects of Alzheimer disease among caregivers, medical students, and healthcare professionals // Neurología. 2021. № 36 (1).

11. Nugraha D., Suyitnno. On the beginning of modern Indonesian literature // Humanities \& Social Sciences Reviews. 2019. № 6. Vol. 7.

12. Schiffrin D. Discourse Markers. Studies in Interactional Sociolinguistics. N. Y.: Cambridge University Press, 1987.

13. Yang Y. An Efficient Polynomial Interior-Point Algorithm for Linear Programming // Journal of the Operations Research Society of China. 2021. № 9.

\section{Информация об авторах | Author information}

RU Некрасова Мария Юрьевна ${ }^{1}$

Миронцева Светлана Сергеевна ${ }^{2}$, к. пед. н.

Байко Валерия Александровна ${ }^{3}$, к. филол. н.

1,2, 3 Севастопольский государственный университет

EN Nekrasova Mariya Yur'evna ${ }^{1}$

Mirontseva Svetlana Sergeevna ${ }^{2}, \mathrm{PhD}$

Baiko Valeriya Aleksandrovna ${ }^{3}, \mathrm{PhD}$

1,2, 3 Sevastopol State University

${ }^{1}$ nekramar@mail.ru, ${ }^{2}$ mirontseva.sse@mail.ru, ${ }^{3}$ beskar_valeria@inbox.ru

\section{Информация о статье | About this article}

Дата поступления рукописи (received): 08.10.2021; опубликовано (published): 30.11.2021.

Ключевые слова (keywords): маркеры аргументации; типы маркеров; научный дискурс; языковые единицы; argumentative markers; types of markers; scientific discourse; linguistic units. 